\title{
Interaction of magnetic elements in metallic ferromagnetic nanofilms
}

\author{
Panaetov V. $P^{I}$.
}

\author{
'. State establishment "High mountainous geophysical institute", Kabardino-Balkar Republic. Nalchik, Lenin Street 360030. 2. \\ Rossiya._email: Valen_pvs@rambler.ru
}

\begin{abstract}
In this paper, a comparative analysis of the distances between magnetic elements is made, under which a noticeable effect arises when they interact. The sides of the domain (domain walls), the terminal part of the domain (domain apex), we called magnetic elements. During magnetization, the domain structure is restructured. The distances between the domain walls, between the domain wall and domain apex, between the apexes of the domains change. A metallic ferromagnetic nanofilm can be a medium for recording information with the help of magnetic moments of electrons. Interaction of domain walls, the interaction of the domain wall and the domain apex, the interaction of the domain apexes in the design of storage devices must be taken into account.
\end{abstract}

\section{The interaction of homogeneous domain walls}

Micro magnetization processes were observed at high magnification in a transmission electron microscope UMV-100K by the method of Lorentz microscopy. The films were obtained by the method described in [1]. In [1] it was shown theoretically and experimentally in which cases the existence of double domain walls in nanofilms with definite magnetic parameters is energetically more advantageous The width of the domain walls changes as the distance between them changes. In [2], double walls with the same and opposite direction of rotation of the magnetization vectors in nanofilms were experimentally proved. It follows from theoretical calculations that for the same direction of rotation of the magnetization vectors in the walls, the width of the walls should decrease with decreasing distance between the walls and increase for walls with the opposite direction of rotation of the magnetization vectors in the walls. By photometry of domain walls at different distances between them, the following graphs of the dependence of the width of the domain walls on the distance between them were obtained. With the same direction of rotation of the magnetization vectors in the walls, the walls are repelled from each other. With an opposite direction of rotation of the magnetization vectors, attraction arises in the walls. These studies are presented in [2-3].

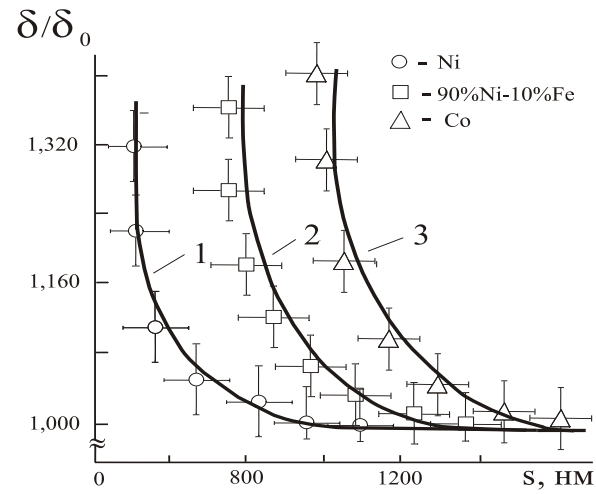

Fig.1. Experimental (O- $\square-\Delta)$ and calculated (solid) curves for the dependence the width domain walls on the distances between them. $\delta$ - The width of the interacting wall. $\delta_{0}-$ The width no interacting wall.

The experimental dependences of $\delta / \delta_{0}$ on the distance $\mathrm{s}$ for nickel films $\left(\mathrm{I}_{\mathrm{s}}=0,48 \mathrm{~A} / \mathrm{m}\right)$, cobalt $\left(\mathrm{I}_{\mathrm{s}}=1,40 \mathrm{~A} / \mathrm{m}\right)$ and $\mathrm{Ni}_{0,90}-\mathrm{Fe}_{0,1}\left(\mathrm{I}_{\mathrm{s}}=0,65 \mathrm{~A} / \mathrm{m}\right)$ are shown in Fig. 1. $\mathrm{I}_{\mathrm{s}}-$ saturation magnetization. The distance between the walls increases with increasing saturation magnetization.

\section{Interaction of periodic domain walls}

Periodic domain walls do not interact with each other in Fig. 2a. This is manifested in the fact that the transverse bonds on the domain walls smoothly transform into the ripple of the magnetization, there are no curvatures of the transverse bonds, there are no bends of the domain walls. If an external magnetic field is applied along the axis of easy magnetization (AEM), it is possible to reduce the distance between the walls so that the transverse bonds of one wall are closed onto the circular lines of the other wall. Such an interaction is shown in Fig. 2b.
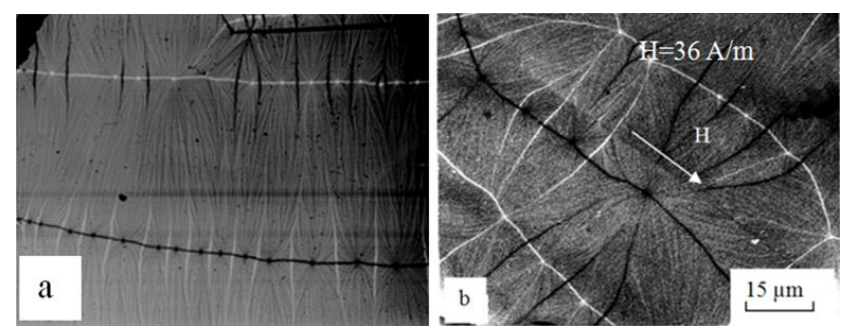

Fig.2. a - Non interacting periodic domain walls. b Interactive periodic domain walls. Composition $\mathrm{Fe}_{0,25}$ $\mathrm{Ni}_{0,75}$, thickness of film $40 \mathrm{~nm}$. Coercive force $\mathrm{H}_{\mathrm{c}}=28$ $\mathrm{A} / \mathrm{m}$, uniaxial anisotropy, $\mathrm{H}_{\mathrm{k}}=160 \mathrm{~A} / \mathrm{m}$.

\section{Interaction of the domain wall with of the domain apex}

On the walls and of domains apexes there is a violation of the continuity conditions for the magnetization 
vectors $I_{\mathrm{s}}\left(\operatorname{div} I_{\mathrm{s}}=0\right)$, then the walls and apices are sources of scattering fields. Therefore, they must interact with each other. When the external magnetic field applied along the AEM is changed, the domain increases or decreases depending on the direction of the field, and the distance between of the domain apex and the domain wall changes. The films were demagnetized before the observation by an alternating magnetic field. Then a DC magnetic field was applied along the axis of easy magnetization (AEM) or the axis of difficult magnetization (ADM) of the film, and either a domain wall displacement process or a magnetization reversal was observed, respectively. Several equilibrium states of a demagnetized film with homogeneous walls $25 \mathrm{~nm}$ in thickness are $\mathrm{Fe}_{0,20}-\mathrm{Ni}_{0,80}$ in Fig. 3, a, b, c. The arrows in the figure indicate areas in which the upper part of the domain and part of the domain wall interact with each other. In the initial state, the domain apex has a weak effect on the wall (Fig. 3a). When a magnetic field $\mathrm{H}$ $<\mathrm{H}_{\mathrm{c}}$ is applied along the AEM, the domain structure as a whole remains unchanged with the exception of the section of the wall located near the apex.

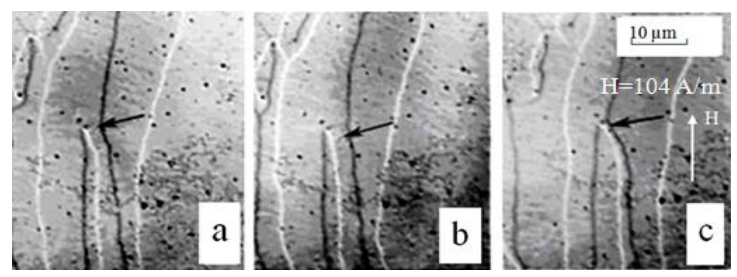

Fig.3. Interaction of domain apex with a domain wall. $\mathrm{H}_{\mathrm{c}}=100 \mathrm{~A} / \mathrm{m}, \mathrm{H}_{\mathrm{k}}=360 \mathrm{~A} / \mathrm{m}$.

The distance between of the domain apexes and the domain wall is reduced. On this section the wall weakly bends toward the apex (Fig. 3, b). When the magnetic field $\mathrm{H}$ is increased to the value of the field $\mathrm{H} \sim$ equal to the field $H_{c},\left(H_{c}\right.$ is the coercive force), the bending of the wall increases noticeably (Fig. 3c), which leads to an increase length of wall and, consequently, its energy.

\section{Interaction of domain apexes in films}

Figure 4a shows a photograph of the magnetic structure of the two apexes from different domains located at a distance $\sim 150 \mu \mathrm{m}$ from each other. The structure of the domain apexes is symmetrical about the axis of the easy magnetization. Domain apexes are located not strictly along the line passing through the apexes under consideration, but there is a displacement along the axis of the difficult magnetization. Under the action of an external magnetic field, applied along the axis of AEM, whose direction coincides with the direction of the magnetization vectors in the domains, the domains apexes begin to approach each other. At a certain distance between domains, a magnetostatic interaction arises. The interaction manifests itself in the fact that the distribution of the magnetization vectors at of the apexes of domains varies, this is noticeable from a change in the ripple of magnetization (or the dispersion of the magnetic anisotropy). In particular, near the apexes of both domains appear domains formed from transverse bonds, in the form of triangles, the magnetization in which is directed along the ADM. Between the apexes there is a domain through which the magnetic flux closes between domains.
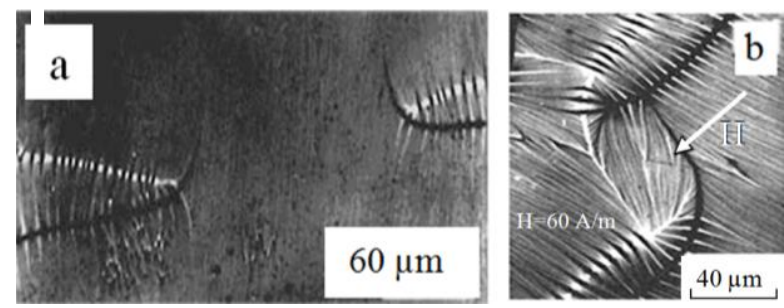

Fig.4. a - non interacting of apexes of domains. b - Ininteracting apexes; thickness $50 \mathrm{~nm}$, composition $\mathrm{Fe}_{0,20^{-}}$ $\mathrm{Ni}_{0,80} . \mathrm{H}_{\mathrm{c}}=40 \mathrm{~A} / \mathrm{m}, \mathrm{H}_{\mathrm{k}}=250 \mathrm{~A} / \mathrm{m}$.

The resulting distribution of the magnetization vectors leads to a decrease in the energy of the scattering fields between the apexes of the domains. The distance between the interacting of the domain apexes is $80 \mu \mathrm{m}$. Further convergence of domain apexes causes their annihilation.

\section{Conclusions}

Many experimental results of the interaction of magnetic elements could not be placed within the scope of this article. It follows from the above results that the greatest distance at which the there is interaction $\sim 100 \mu \mathrm{m}$ is the interaction between of apexes of domains. Between the periodic walls, the interaction manifests itself at distances between them of $\sim 20-30 \mu \mathrm{m}$. Between the homogeneous walls, the interaction is manifested at distances of $\sim 1-2 \mu \mathrm{m}$. All these results depend on the specific magnetic parameters of the film. Such parameters are saturation magnetization, perpendicular anisotropy constant, film thickness, etc.

\section{References}

1. V. P. P a n a e tov. JNTM Vol. 05, $-\mathrm{N}^{\circ} 02$ (2015)

2. V. P. Panaetov. Physics of the Solid State, , Vol. 51, No. 10 (2009)

3 .V.P. Panatov. Bulletin of Higher Educational Institutions Vol. 60 №2 (2) 017 ) 